EXTENDED REPORT

\title{
Histopathological and immunohistochemical studies of lenticules after epikeratoplasty for keratoconus
}

\author{
H Nakamura, F Riley, H Sakai, W Rademaker, B Y J T Yue, D P Edward
}

Br J Ophthalmol 2005;89:841-846. doi: 10.1136/bjo.2004.054684

\section{See end of article for authors' affiliations \\ Correspondence to: Deepak P Edward, University of Illinois at Chicago, Department of Ophthalmology and Visual Sciences, 1855 W Taylor Street, Chicago, IL 60612, USA; deepedwa@uic.edu}

Accepted for publication 1 December 2004

\begin{abstract}
Aims: To examine histopathological and immunohistochemical changes in lenticules and host of corneal buttons from patients who previously underwent epikeratoplasty for keratoconus.

Methods: 12 penetrating keratoplasty specimens from patients with keratoconus who had previously undergone epikeratoplasty, eight keratoconus, and seven normal corneas were examined. Immunostaining for Spl, $\alpha 1$-proteinase inhibitor ( $\alpha 1-\mathrm{PI})$, and $\alpha 2$-macroglobulin $(\alpha 2 \mathrm{M})$ were performed. Results: In nine of the 12 lenticules, the keratoconus-like disruptions were found in Bowman's layer. Peripheral and posterior keratocyte repopulation of the lenticules was observed in all cases. Keratocyte repopulation in the anterior and mid-stromal regions of the lenticules appeared related to the time since epikeratoplasty. Spl nuclear staining of the basal and wing epithelial cells was more intense in lenticules and keratoconus corneas than in normal corneas. Lenticular, host, and keratoconus keratocytes showed positive Spl staining, whereas staining was absent in normal corneas. Compared to normal corneas, $\alpha 1$ $\mathrm{Pl}$ and $\alpha 2 \mathrm{M}$ immunostaining was lower in the lenticules, host, and keratoconus specimens.

Conclusions: The epithelial cells and keratocytes repopulated in the lenticules retain keratoconus-like biochemical abnormalities such as upregulation of Spl and downregulation of $\alpha 1-\mathrm{PI}$ and $\alpha 2 \mathrm{M}$. The authors speculate that both keratocytes and the corneal epithelium may participate in the development of keratoconus.
\end{abstract}

$\mathrm{E}$ pikeratoplasty is a form of lamellar refractive corneal surgery introduced in $1980 .{ }^{1}$ The surgical procedure was first used to correct aphakia (epikeratophakia), ${ }^{1-3}$ and was then adapted for the treatment of myopia. ${ }^{4}$ In this procedure, the recipient cornea is denuded of epithelium, and the edges of a preshaped donor lenticule are sutured to an annular keratectomy in the peripheral host cornea. ${ }^{5}$ Epikeratoplasty has also been a surgical option for treatment of keratoconus ${ }^{6}{ }^{7}$ for instance, for patients who engaged in strenuous physical activity ${ }^{8}$ or in regions, such as Saudi Arabia, where patients do not have easy access to tertiary care facilities. ${ }^{9}$

Keratoconus, a non-inflammatory disease, is characterised by disruption of Bowman's layer, ${ }^{10-12}$ thinning and scarring of the stroma, and the eventual protrusion of the central cornea. ${ }^{13}$ Immunohistochemical and biochemical studies have shown that the defect of keratoconus may involve an abnormality in degradation processes. ${ }^{14}{ }^{15}$ Enzymes, such as cathepsin $\mathrm{B}$, are elevated, and protease inhibitors such as $\alpha \mathrm{l}$ proteinase inhibitor ( $\alpha$ l-PI) and $\alpha 2$-macroglobulin $(\alpha 2 \mathrm{M})$ are downregulated in keratoconus corneas. ${ }^{16-18}$ Furthermore, our laboratory has reported that Spl, a ubiquitous transcription factor, is upregulated in the corneas of keratoconus patients. ${ }^{19}$ In cultured human corneal fibroblasts and epithelial cells overexpression of $\mathrm{Spl}$ has been shown to reppress the promoter activity of the $\alpha 1-P I$ gene. ${ }^{20}{ }^{21}$ This suggests that the Spl-mediated downregulation of the $\alpha 1$-PI gene may be a key event leading to the increased degradation and pathology in keratoconus corneas.

Histopathological analyses of failed epikeratoplasty lenticules for keratoconus and other corneal disease have been previously described. ${ }^{22-30}$ In those reports, Bowman's layer abnormalities in lenticules, including bends, ${ }^{23} 24{ }^{27}$ breaks, $^{24}$ and absences, ${ }^{24} 2528$ were identified, and the keratocyte repopulation into lenticules was noted to be more frequent in the anterior than the posterior region of central lenticules. $^{22} 2329$ However, the reported abnormalities were unlike those observed in keratoconus corneas. These included large defects of Bowman's layer that were believed to result from long term epithelial defects. These breaks were dissimilar to the focal " $\mathrm{z}$ "-shaped disruptions in Bowman's layer or absence of small segments of Bowman's layer more typically seen in keratoconus corneas. ${ }^{1031}$ Such keratoconus-like fractures in Bowman's layer were noted in the recipient grafts of recurrent keratoconus after penetrating keratoplasty. ${ }^{32-36}$ Recurrent cases of keratoconus are rare and immunohistochemical and/or biochemical investigation of the grafts were not reported.

In this study we evaluated the histopathological and immunohistochemical changes in 12 lenticules from patients who previously underwent epikeratoplasty for keratoconus. We investigated the integrity of Bowman's layer of the lenticules, keratocyte repopulation in lenticules, and biochemical changes in the epithelium and stroma of both lenticules and host corneas. We found that the grafted lenticules displayed abnormalities similar to those found in keratoconus corneas.

\section{MATERIALS AND METHODS}

Twelve corneal buttons were obtained from patients who had previously received epikeratoplasty for management of keratoconus at the time of penetrating keratoplasty from the King Khaled Eye Specialist Hospital, Riyadh. The grafts were done because of varying degrees of uncorrected refractive problems following the epikeratoplasty. Seven normal human eyes from donors (ages 22-83 years old) were obtained from the Illinois Eye Bank, Chicago, or from the National Disease Research Interchange, Philadelphia, PA, within 24 hours of death. None of the donors had any known ocular diseases, and their corneas were clear and unremarkable. As another set of controls, eight corneal buttons from

Abbreviations: $\alpha 1-\mathrm{Pl}, \alpha 1$-proteinase inhibitor; $\alpha 2 \mathrm{M}, \alpha 2$-macroglobulin; $\mathrm{ECM}$, extracellular matrix 


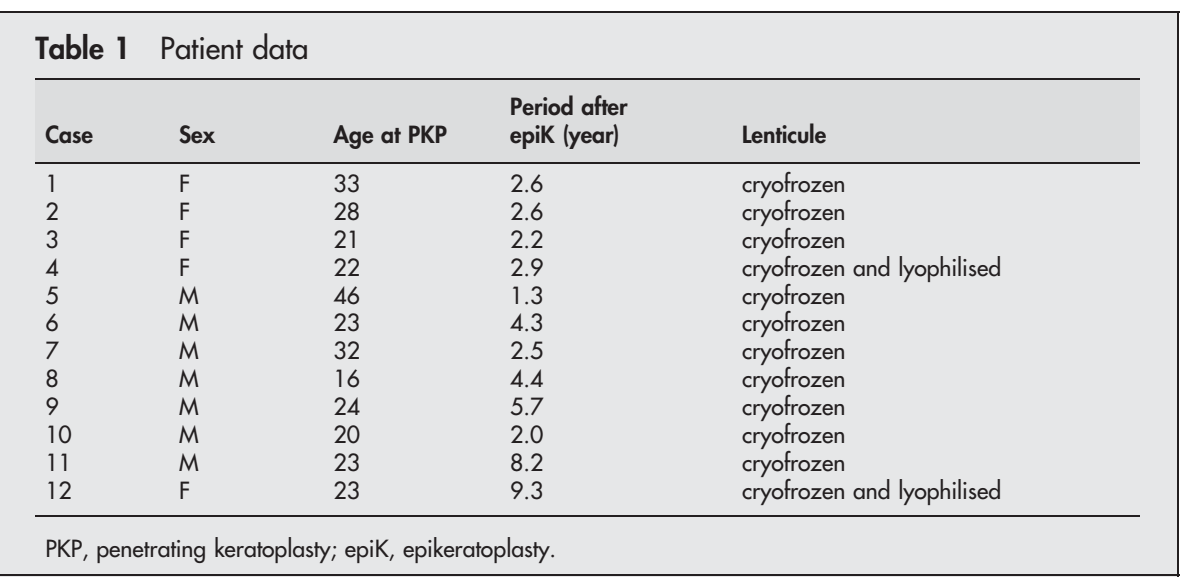

patients (ages 22-70 years) with typical clinical features of keratoconus but without undergoing epikeratoplasty surgery previously were obtained following transplantation from the Cornea Service at the University of Illinois at Chicago.

Corneas excised from normal human eyes and keratoconus buttons were fixed in $10 \%$ buffered formalin, processed, and embedded in paraffin. Immunohistochemistry was performed on deparaffinised $5 \mu \mathrm{m}$ sections using the indirect immunoperoxidase technique. The primary antibodies used in the study included (a) a polyclonal rabbit anti-Spl antibody (PEP 2, diluted 1:100, Santa Cruz Biotechnology, Santa Cruz, CA, USA), (b) polyclonal goat antibodies specific for $\alpha$ l-PI (1:100, ICN Biomedicals, Irvine, CA, USA), and (c) $\alpha 2 \mathrm{M}$ (1:100, ICN Biomedicals). The chromogen used for the anti-Spl was fast red TR/naphthol AS-MX phosphate (Sigma, St Louis, MO, USA). For $\alpha 1$-PI and $\alpha 2 \mathrm{M}, 3,3$-diaminobenzidine tetrahydrochloride (Sigma) was used as the chromogen. The staining intensity in each experiment was scored by three masked observers on a scale of 0 to 4 , with 0 indicating no staining and 4 the most intense staining. Experiments were repeated three times.

Histopathological changes in the epikeratoplasty specimens were evaluated on haematoxylin and eosin stained slides. Alterations in Bowman's layer of the lenticules were evaluated only in the central regions of the specimens to exclude breaks seen along suture tracks in the peripheral regions of the specimens.

To confirm the labelling intensity evaluated by the masked observers we performed image analysis on the selected images presented in the paper demonstrating imunostaining with the three antibodies (see figs 3, 5, and 6). The image file was converted from RGB to grey scale image using Adobe Photoshop 7.01. Imaging Processing Tool kit 3.0 (Reindeer Games, Inc), incorporated in the Adobe Photoshop 7.01, was used to measure the intensity of staining. The intensity, judged as 256 shades of grey with 0 representing black and 256 representing white, was measured in at least six basal epithelial cells, wing cells, keratocytes, and/or six areas (25 $\mu \mathrm{m}$ diameter circle) in the stromal matrix. Background intensity was also taken in the empty space. The staining intensity of each cell type or the stromal matrix tissue was obtained by subtracting the background from the measured intensity values. Statistical analysis was performed using two tailed unpaired Student's $t$ test to compare the staining intensity of KC or EpiK cornea with normal control. Values of $\mathrm{p}<0.05$ was considered to be significant.

\section{RESULTS}

The salient clinical features of the 12 patients who had previously received epikeratoplasty as a treatment for keratoconus are summarised in table 1 .

By histopathology, the keratoconus-like breaks in Bowman's layer were found in nine of the 12 failed lenticules (table 2, fig 1).

The presence or the number of the breaks was not correlated with either the sex or the length of period since epikeratoplasty.

Keratocyte repopulation was observed in all lenticules at all levels of the peripheral stroma (table 2). Keratocytes were identified morphologically by the spindle-shaped nature of their nuclei, which are quite distinct from other cells such as leucocytes that might be resident in the corneal stroma. The

\begin{tabular}{|c|c|c|c|c|c|c|c|}
\hline \multirow[b]{3}{*}{ Case } & \multirow{3}{*}{$\begin{array}{l}\text { Bowman's } \\
\text { layer } \\
\text { disruption }\end{array}$} & \multicolumn{6}{|c|}{ Keratocyte repopulation } \\
\hline & & \multicolumn{3}{|c|}{ Centre } & \multicolumn{3}{|c|}{ Periphery } \\
\hline & & A & M & $\mathbf{P}$ & A & M & $\mathbf{P}$ \\
\hline 1 & + & + & + & + & + & + & + \\
\hline 2 & + & & & + & + & + & + \\
\hline 3 & & & & + & + & + & + \\
\hline 4 & & & & + & ++ & + & + \\
\hline 5 & + & & & + & + & + & + \\
\hline 6 & + & + & + & + & + & + & + \\
\hline 7 & + & & & + & + & + & + \\
\hline 8 & & + & + & + & + & + & + \\
\hline 9 & + & & & + & H & + & + \\
\hline 10 & + & & & + & + & + & + \\
\hline 11 & + & + & + & + & ++ & ++ & + \\
\hline 12 & + & + & + & + & ++ & + & + \\
\hline
\end{tabular}



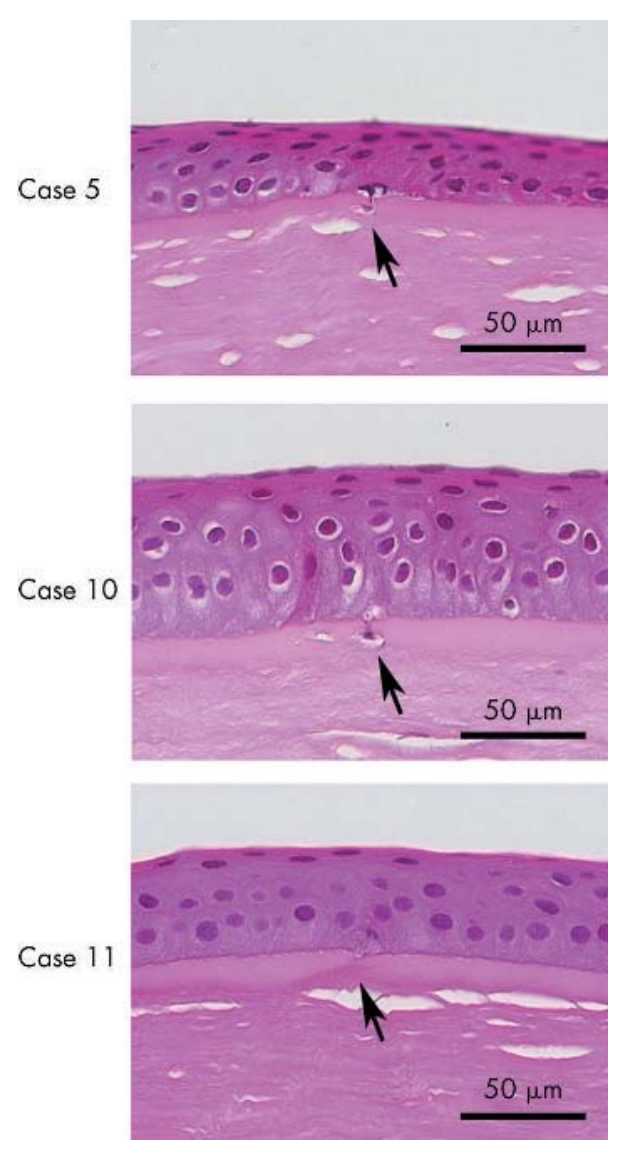

Figure 1 Lenticules from patients who underwent epikeratoplasty for keratoconus (cases 5, 10, and 11). Arrow indicates the focal disruption in Bowman's layer. The disruption or break resembles that described in keratoconus corneas (bar indicates magnification).

cells with spindle-shaped nuclei accounted for most of the cells within the lenticules. Rare cells consistent with leucocytes in the stroma were also noted. In the central region, repopulation was also observed in all lenticules in the posterior portion. Keratocytes that repopulated the posterior portion of the lenticules were mainly seen adjacent to Bowman's layer of the host corneas. In some areas, keratocytes appeared to migrate through the Bowman's break of the host tissues (fig 2). However, repopulation in the anterior and mid-stromal region of the central lenticule was noted in only five lenticules. In these cases, the patients had a significantly longer history of epikeraptolasty than the others ( 5.8 (SD 2.8) v 2.7 (1.4) years; $\mathrm{p}<0.05$, Mann-Whitney $\mathrm{U}$ test, tables 1 and 2 ).

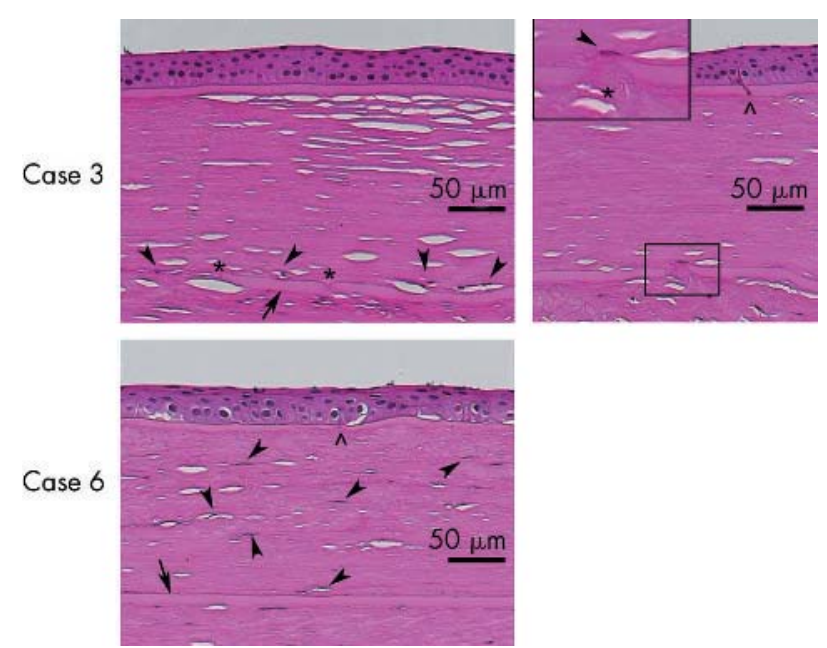

Figure 2 The central portion of the lenticules and host corneas from cases 3 and 6. Arrow indicates Bowman's layer of the host cornea. Arrowheads indicate keratocytes repopulated in the lenticules. Asterisk denotes a break observed in Bowman's layer of host. The symbol $\wedge$ indicates disruption of Bowman's layer in the lenticule. In case 3, a spindle cell appeared to extend through the break in Bowman's layer from the host stroma to the lenticule. The repopulated keratocytes were situated adjacent and right above the host Bowman's layer. In case 6, keratocyte repopulation was observed throughout the anterior and posterior regions of the lenticules (bar indicates magnification).

Immunohistochemical experiments showed positive Spl staining in the nuclei of basal corneal epithelial cells and wing cells in the lenticules, as well as keratocytes in both the lenticules and host stromas. The staining intensity in the epithelial cells of lenticules and that in the stromal cells in the lenticules and host corneas were statistically higher than that in normal human corneas $(\mathrm{p}<0.05$, figs 3 and 4$)$. Keratoconus corneas, used as positive controls, also showed strong nuclear staining of comparable intensity for Spl in both corneal epithelial cells and keratocytes.

Compared to that in normal human corneas, immunostaining for both $\alpha \mathrm{l}$-PI and $\alpha 2 \mathrm{M}$ was considerably weaker in epithelial cells of the lenticules, and keratocytes in the lenticules and host stromas (figs 5 and 6). Each of these differences was statistically significant $(\mathrm{p}<0.05$, figs 7 and 8$)$. A similar reduction in staining intensity was likewise observed in keratoconus corneas. Staining intensity in the stromal lamellae for $\alpha$ l-PI in the lenticules and host corneas was also statistically lower than that in normal corneas $(\mathrm{p}<0.05$, figs 6 and 7$)$. The staining for $\alpha 2 \mathrm{M}$ was also reduced but the difference was not statistically significant from the normal controls (figs 6 and 8). In the epikeratoplasty specimens the immunoreactivity with three antibodies in
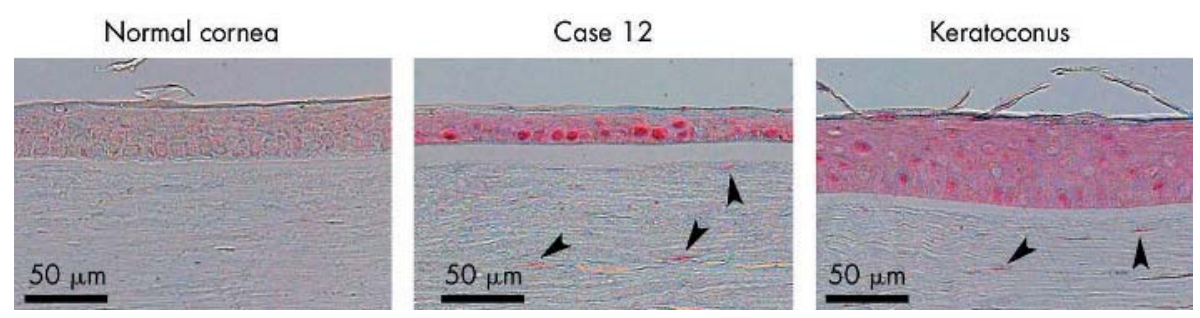

Figure 3 Immunostaining for Spl in corneas from a 61 year old normal individual and a 22 year old keratoconus patient, as well as a lenticule of a patient after epikeratoplasty for keratoconus (case 12). In both the lenticule and keratoconus corneas (positive controls), the nuclear immunolabelling was stronger in basal corneal epithelial cells, wing cells, and keratocytes than cells in the normal cornea (chromagen fast red TR/naphthol AS-MX phosphate). Image analysis confirmed significant differences in labelling intensity between normal control (epithelium: basal: 35.8 (SD 4); wing: 32 (4); stromal cell: 9 (3)) and epikeratoplasty (epithelium, basal: 64 (7) ( $p=0.000008)$; wing: 59 (8) ( $p=0.001)$; stromal cell: 82 (4) ( $p=0.00001))$, and keratoconus (epithelium: basal: 55 (4) ( $p=0 / 001)$; wing: 55 (3) ( $<<0.00001)$; stromal cell: 81 (2) $(p=<0.00001)$ ). 


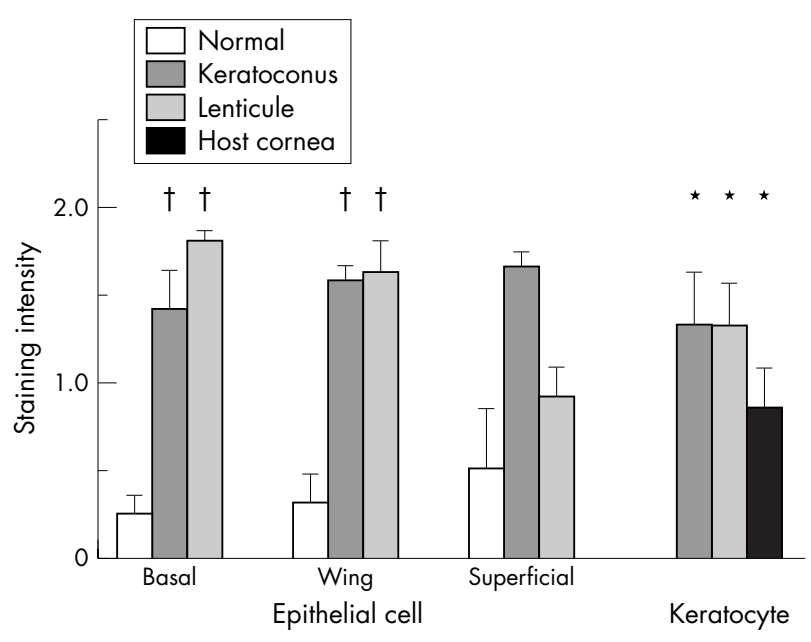

Figure 4 Staining intensity for $\mathrm{Sp} 1$ in the corneal epithelial cells and keratocytes in the lenticules $(n=12)$, host stromas $(n=12)$, and normal human $(n=4)$ and keratoconus $(n=3)$ corneas as scored by three masked observers. The scores were analysed by Mann-Whitney $\mathrm{U}$ tests. ${ }^{*} p<0.01$ compared with normal human specimens; $\uparrow p<0.05$.

the lenticular stroma and the host was comparable (figs 6, 7, and 8). As expected, the staining intensity was comparable in the keratoconus and epikeratoplasty specimens.

Image analysis confirmed the staining differences that were observed on semiquantitative assessment of labelling intensity (see fig 3, 5, and 6)

\section{DISCUSSION}

To the best of our knowledge, this is the first demonstration of keratoconus-like breaks in Bowman's layer in lenticules from eyes that previously underwent epikeratoplasty as a treatment for keratoconus. The breaks observed in our study occurred with or without keratocyte repopulation and closely resembled those described previously in keratoconus. Both the presence of Bowman's breaks and absence of Bowman's layer have been reported previously in lenticules after epikeratoplasty for myopia, ${ }^{24} 25$ aphakia, ${ }^{24} 28$ and keratoconus. ${ }^{28}$ However, those large breaks, at times with almost total loss of Bowman's layer, differed from the focal breaks or fragmentation observed in our cases in the failed lenticules and in typical keratoconus corneas. ${ }^{10-12}$ Absence of large fragments of Bowman's layer is unusual in typical keratoconus and if seen may be associated with hydrops or associated contact lens related complications. The incidence of Bowman's layer disruption was also much higher in our cases (nine of 12 cases) than that in previous reports (eight of a total of 21 cases in the literature). ${ }^{22-28}$

This is also the first demonstration of remarkable keratocyte repopulation of the posterior stroma in the central lenticule. Keratocyte repopulation in lenticule had been reported to be slow process from peripheral region, and to take 48 months to complete. ${ }^{30}$ In our cases, the central posterior repopulation was noted in all 12 cases examined including seven lenticules which were examined at the time of less than 4 years after epikeratoplasty (fig 2 and table 2 ). Since all of the lenticules in this series were cryofrozen before the epikeratoplasty, the donor keratocytes would not be expected to survive through the cryofreezing process. ${ }^{37} 38$ Therefore, we suggest that the cells present in the lenticules were host keratocytes, and that the keratocytes in the posterior region of the lenticule migrated from underlying host tissue through breaks in the host Bowman's layer.

As previously reported in eyes with and without keratoconus, 22232930 we noted that keratocytes repopulated the lenticules in the periphery, and that keratocyte repopulation was not predominant in the superficial and central region of the lenticules (table 2). In five lenticules, repopulation in the anterior or mid-stromal portions of central region was observed and the extent of keratocyte repopulation in these regions, interestingly, seemed to correlate with the length of time since epikeratoplasty. The time dependence supports a previous conclusion ${ }^{30}$ that the anterior and middle lenticular keratocyte repopulation perhaps takes place gradually from the periphery, along stromal lamella.

Immunohistochemical experiments demonstrated that in the epithelium overlying the lenticules, Spl staining was increased and staining for inhibitors $\alpha \mathrm{l}-\mathrm{PI}$ and $\alpha 2 \mathrm{M}$ was decreased. These have been shown to be changes that are specific to keratoconus corneas. ${ }^{16-19}$ The epithelium of the lenticules thus displayed a keratoconus-like phenotype, which is not surprising as the epithelium was derived from
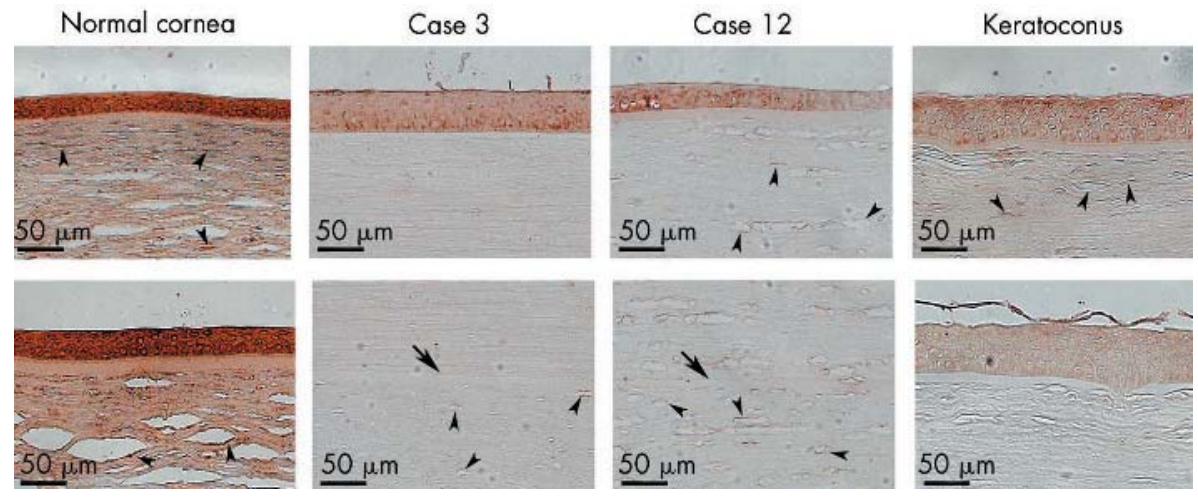

Figure 5 Immunostaining for $\alpha 1$-proteinase inhibitor in corneas from 83 year old (top) and 78 year old (bottom) normal individuals, and a 70 year old keratoconus patient, as well as lenticules of patients after epikeratoplasty for keratoconus (cases 3 and 12). Arrow indicates Bowman's layer of the host cornea. Arrowheads indicate keratocytes. Note that the intensity of the brown positive staining is lower in the epithelium, keratocytes, and stromal lamellae in the lenticules and host corneas than that in normal corneas. Staining is also weaker in keratoconus corneas compared to normal controls (chromagen 3-3' diaminobenzidine). Image analysis confirmed significant differences in labelling intensity between normal control (epithelium: basal: 153.5 (3); wing: 141 (3); stromal cell: 179 (3); stromal extracellular matrix (ECM) 72 (5)) and epikeratoplasty case 3 (epithelium: basal: 63 (8) ( $p<0.0000001)$; wing: 53 (4) ( $p<0.000001)$; stromal cell in lenticule: 31 (6) ( $p=0.000001)$, stromal ECM in lenticule: 14 (2) ( $<<0.000001)$; stromal cell in host: 19 (2) $(p<0.00001)$, stromal ECM in host: 12 (1) ( $<<00001)$ ) and keratoconus (epithelium: basal: 43 (4) ( $<<0.0000001)$; wing: 49.3 (10) $(p<0.00000001)$; stromal cell: 37 (8) ( $<<0.000001)$; stromal ECM 12.5 (3) $(p<0.00001))$. Image analysis for case 12 also showed highly significant differences from control. 

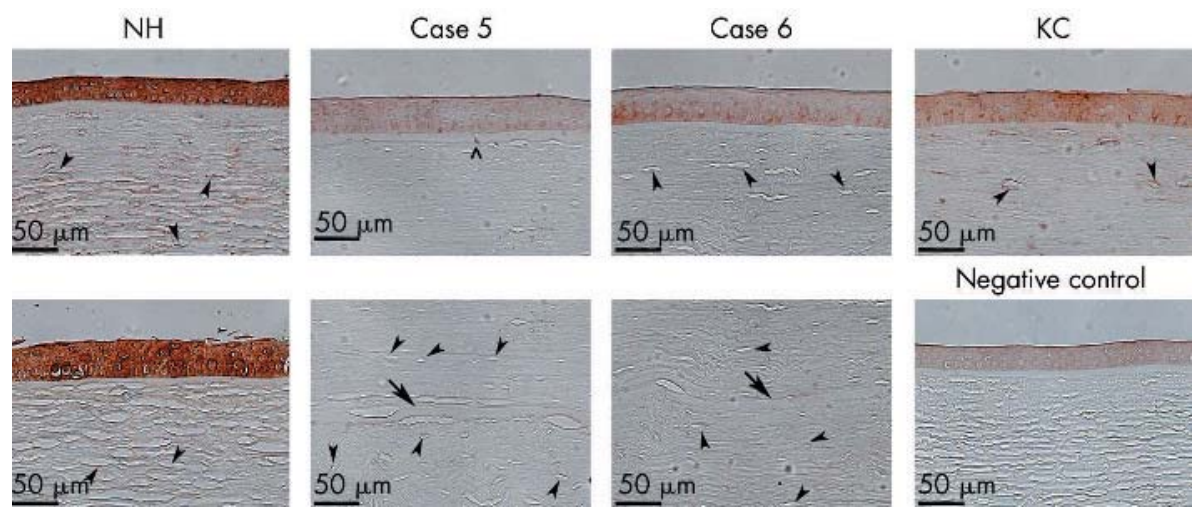

Figure 6 Immunostaining for $\alpha 2$-macroglobulin in corneas from 83 year old (top) and 22 year old (bottom) normal individuals (NH), lenticules of patients affer epikeratoplasty for keratoconus (cases 5 and 6 ), and a cornea from a 70 year old keratoconus patient (KC). Arrow indicates Bowman's layer of the host cornea. Arrowheads indicate keratocytes. The symbol $\wedge$ indicates disruption of Bowman's layer in the lenticule. Note that the intensity of the brown positive staining is decreased in the epithelium, keratocytes, and stromal lamellae in the lenticules, host corneas, and keratoconus specimens when compared to the normal corneas (chromogen 3-3' diaminobenzidine). Image analysis confirmed significant differences in labelling intensity between normal control (epithelium: basal: 91 (2); wing: 98 (6); stroma cell: 51 (7); stromal ECM: 12 (1)); epikeratoplasty (case 5) (epithelium: basal: 29 (9) ( $p<0.00009)$; wing: 27 (4) ( $p=0.00003)$; stromal cell in lenticule: 11 (13) ( $p=0.0001)$; stromal ECM in lenticule: 5 (1) ( $<<0.0001)$; stromal cell in host: 15 (18) ( $p=0.001)$; stromal ECM in host: 1.8 (2) ( $p<0.00004))$ and keratoconus (epithelium: basal: 52 (5) ( $<<0.0000003)$; wing: 55 (5) ( $<<0.00000004)$; stromal cell:52 (14) $p=0.90$; stromal ECM: 10 (1) $(p=0.03)$.

the host. The role of the corneal epithelium in development of keratoconus was proposed in the $1960 \mathrm{~s}^{10} 1139$ based on electron microscopic studies. Further evidence was provided when biochemical abnormalities including upregulation of degradative enzymes and downregulation of inhibitors were prominently observed in the keratoconus epithelium. ${ }^{16-18} \mathrm{~A}$ study by three dimensional scanning electron microscopy ${ }^{12}$ further indicated that the changes in the Bowman's layer precede those in the corneal stroma and that the corneal epithelium may be an important factor at the early stage of keratoconus development. The current finding of alterations in the epithelium overlying the lenticules is consistent with this theory. It is possible that the corneal epithelium overlying the lenticules, with imbalanced enzyme and inhibitor levels, may cause disruption in Bowman's layer typically seen in keratoconus specimens.
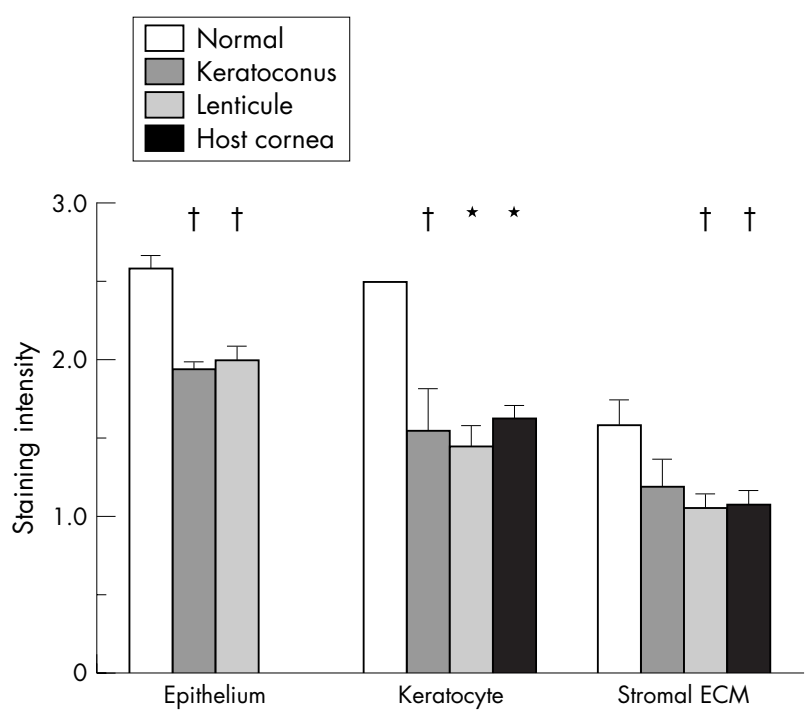

Figure 7 Staining intensity for $\alpha 1$-proteinase inhibitor in corneal epithelial cells, keratocytes, and stromal extracellular matrix (ECM) in the lenticules, host stromas, and normal human and keratoconus corneas as scored by three masked observers. The scores were analysed by MannWhitney $U$ tests. ${ }^{*} \mathrm{p}<0.01$ compared with normal human specimens; $\mathrm{tp}<0.05$.
In the lenticular stroma, upregulation of Spl was demonstrated in the repopulated keratocytes. Downregulation of $\alpha \mathrm{l}$ PI and $\alpha 2 \mathrm{M}$ was also observed. These results indicate that the repopulated keratocytes retained the biochemical abnormalities ascribed to keratoconus. The rarity of recurrent cases of keratoconus after penetrating keratoplasty seems to argue against the corneal epithelial theory. Indeed, if the epithelial cells were the sole causative factor, ${ }^{10}{ }^{11}{ }^{39}$ frequent recurrence of keratoconus would occur as the epithelial cells are expected around 5 days to migrate from the recipient into the donor cornea. ${ }^{7}$ It is possible that the corneal epithelial involvement is necessary but not sufficient. We speculate that perhaps both the disruption of the Bowman's layer ${ }^{10} 111239$ and a predisposed defect in keratocytes are required for the disease development. The current study, showing defects in Bowman's layer, delayed keratocyte repopulation, and biochemical abnormalities ascribed to keratoconus in the repopulated keratocytes in failed lenticules 15 months to 9 years after epikeratoplasty, supports this possibility.

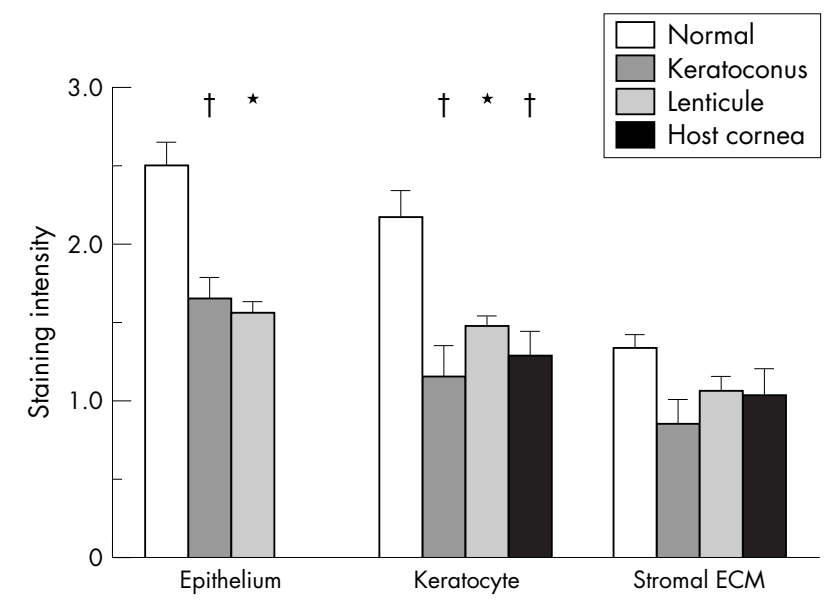

Figure 8 Staining intensity for $\alpha 2$-macroglobulin in corneal epithelial cells, keratocytes, and stromal extracellular matrix (ECM) in the lenticules, host stromas, and normal human and keratoconus corneas as scored by three masked observers. The scores were analysed by MannWhitney U tests. ${ }^{*} \mathrm{p}<0.01$ compared with normal human specimens; $+\mathrm{p}<0.05$. 
In summary, we demonstrated keratoconus-like disruptions in Bowman's layer in nine of the 12 lenticules. The keratocytes appeared to repopulate the lenticules from the peripheral and underlying host tissues. By immunohistochemistry, Spl nuclear staining in both epithelium and stroma was increased in the lenticules and keratoconus than that in normal corneas. In contrast, immunostaining for $\alpha \mathrm{l}$ PI and $\alpha 2 \mathrm{M}$ in epithelial cells, keratocytes, and stromal lamellae was less intense in the lenticule, host corneas, and keratoconus specimens than in normal cornea. These findings suggested that the epithelial cells and keratocytes that repopulated the lenticules retained keratoconus-like biochemical abnormalities resulting in some of the changes noted in the lenticules.

\section{Authors' affiliations}

H Nakamura, H Sakai, B Y J T Yue, D P Edward, Department of Ophthalmology and Visual Sciences, University of Illinois at Chicago College of Medicine, Chicago, IL, USA

F Riley, W Rademaker, D P Edward, King Khaled Eye Specialist Hospital, Riyadh, Saudi Arabia

Grant supports: William Pendill Research Fund; EY 03890 (BYJTY), EY 05628 (BYJTY) and EY01792 (core) from the National Eye Institute.

Competing interests: none declared

\section{REFERENCES}

1 Kaufman HE. The correction of aphakia. XXXVI Edward Jackson Memorial Lecture. Am J Ophthalmol 1980;89:1-10.

2 Werblin TP, Kaufman HE, Friedlander $\mathrm{MH}$, et al. Epikeratophakia: the surgical correction of aphakia. III. Preliminary results of a prospective clinical trial. Arch Ophthalmol 1981;99:1957-60.

3 McDonald MB, Kaufman HE, Aquavella JV, et al. The nationwide study of epikeratophakia for aphakia in adults. Am J Ophthalmol 1987:103:358-65.

4 McDonald MB, Kaufman HE, Aquavella JV, et al. The nationwide study of epikeratophakia for myopia. Am J Ophthalmol 1987;103:375-83.

5 Waring GO 3rd. aking sense of 'keratospeak'. A classification of refractive corneal surgery. Arch Ophthalmol 1985;103:1472-7.

6 Kaufman HE, Werblin TP. Epikeratophakia for the treatment of keratoconus. Am J Ophthalmol 1982;93:342-7.

7 McDonald MB, Kaufman HE, Durrie DS, et al. Epikeratophakia for keratoconus. The nationwide study. Arch Ophthalmol 1986;104:1294-300.

8 Goosey JD, Prager TC, Goosey CB, et al. A comparison of penetrating keratoplasty to epikeratoplasty in the surgical management of keratoconus. Am J Ophthalmol 1991;112:103-5.

9 Wagoner MD, Smith SD, Rademaker WJ, et al. Penetrating keratoplasty vs epikeratoplasty for the surgical treatment of keratoconus. J Refract Surg 2001;17:138-46.

$10 \mathrm{Chi} \mathrm{H}$, Katzin H, Teng C. Histopathology of keratoconus. Am J Ophthalmol 1956;42:847-60.

11 Teng CC. Electron microscopic study of pathology of keratoconus. Am J Ophthalmol 1963:55:18-47.

12 Sawaguchi S, Fukuchi $\mathrm{T}$, Abe $\mathrm{H}$, et al. Three-dimensional scanning electron microscopic study of keratoconus corneas. Arch Ophthalmol 1998; 1 16:62-8

13 Rabinowitz YS. Keratoconus. Surv Ophthalmol 1998;42:297-319.
14 Yue BYJT, Sugar J, Benveniste K. Heterogeneity in keratoconus: possible biochemical basis. Proc Soc Exp Biol Med 1984;175:336-41.

15 Yue BYJT, Sugar J, Benveniste K. RNA metabolism in cultures of corneal stromal cells from patients with keratoconus. Proc Soc Exp Biol Med 1985; 178:126-32

16 Sawaguchi S, Twining SS, Yue BY, et al. Alpha-1 proteinase inhibitor levels in keratoconus. Exp Eye Res 1990;50:549-54.

17 Whitelock RB, Fukuchi T, Zhou L, et al. Cathepsin G, acid phosphatase, and alpha 1-proteinase inhibitor messenger RNA levels in keratoconus corneas. Invest Ophthalmol Vis Sci 1997:38:529-34.

18 Sawaguchi S, Twining SS, Yue BY, et al. Alpha 2-macroglobulin levels in normal human and keratoconus corneas. Invest Ophthalmol Vis Sci 1994;35:4008-14.

19 Whitelock RB, Li Y, Zhou L, et al. Expression of transcription factors in keratoconus, a cornea-thinning disease. Biochem Biophys Res Commun 1997;235:253-8.

20 Li Y, Zhou L, Twining SS, et al. Involvement of Spl elements in the promoter activity of the $\alpha 1$-proteinase inhibitor gene. J Biol Chem 1998;273:9959-65.

21 Maruyama Y, Wang X, Li Y, et al. Involvement of Spl elements in the promoter activity of genes affected in keratoconus. Invest Ophthalmol Vis Sci 2001;42:1980-5.

22 Baumgartner SD, Binder PS. Refractive keratoplasty. Histopathology of clinical specimens. Ophthalmology 1985;92:1606-15.

23 Binder PS, Baumgartner SD, Fogle JA. Histopathology of a case of epikeratophakia (aphakic epikeratoplasty). Arch Ophthalmol 1985; 103:1357-63.

24 Binder PS, Zavala EY. Why do some epikeratoplasties fail? Arch Ophthalmol 1987; 105:63-9.

25 Bechara SJ, Grossniklaus HE, Waring GO 3rd. ubepithelial fibrosis after myopic epikeratoplasty. Report of a case. Arch Ophthalmol 1992;110:228-32.

26 Morgan KS, Beverman RW. Interface opacities in epikeratophakia. Arch Ophthalmol 1986;104:1505-8

27 Tamaki K, Yamaguchi T, McDonald MB, et al. Histological study of epikeratophakia tissue lenses for myopia removed from two patients. Ophthalmology 1986;93:1502-8.

28 Frangieh GT, Kenyon KR, Wagoner MD, et al. Epithelial abnormalities and sterile ulceration of epikeratoplasty grafts. Ophthalmology 1988;95:213-27.

29 Grossniklaus HE, Lass JH, Jacobs G, et al. Light microscopic and ultrastructural findings in failed epikeratoplasty. Refract Corneal Surg 1989;5:296-301

30 Yoon YD, Waring GO 3rd, Stulting RD, et al. eratocyte repopulation in epikeratoplasty specimens. Cornea 1998;17:180-4.

31 Feder RS. Noninflammatory ectatic disorders. In: Krachmer JH, Mannis MJ, Holland EJ, eds. Cornea. St Louis, Missouri: Mosby, 1997:1091-106.

32 Abelson MB, Collin HB, Gillette TE, et al. Recurrent keratoconus after keratoplasty. Am J Ophthalmol 1980:90:672-6.

33 Nirankari VS, Karesh J, Bastion F, et al. Recurrence of keratoconus in donor cornea 22 years after successful keratoplasty. $\mathrm{Br} J$ Ophthalmol 1983;67:23-8.

34 Bechrakis N, Blom ML, Stark WJ, et al. Recurrent keratoconus. Cornea 1994;13:73-7.

35 Kremer I, Eagle RC, Rapuano CJ, et al. Histologic evidence of recurrent keratoconus seven years after keratoplasty. Am J Ophthalmol 1995; 119:51 1-12.

36 Bourges JL, Savoldelli M, Dighiero P, et al. Recurrence of keratoconus characteristics: a clinical and histologic follow-up analysis of donor grafts. Ophthalmology 2003;110:1920-5.

37 Rich LF, Friedlander MH, Kaufman HE, et al. Keratocyte survival in keratophakia lenticules. Arch Ophthalmol 1981;99:677-80.

38 Zavala EY, Binder PS, Deg JK, et al. Refractive keratoplasty: lathing and cryopreservation. CLAO J 1985;11:155-62.

39 Iwamoto T, Devoe AG. Particulate structures in keratoconus. Arch Ophthalmol Rev Gen Ophtalmol 1975;35:65-76. 